\title{
SPECTROMÉTRIE DES ÉLECTRONS AUGER
}

\author{
C. BURGGRAF, B. CARRIÈrE et S. GOLDSZTAUB
}

Laboratoire de Minéralogie de Strasbourg, U. L. P. Strasbourg, France

\begin{abstract}
Résumé. - La spectrométrie des électrons Auger permet de caractériser les atomes situés à la surface d'un corps cristallin ou amorphe et de préciser la nature de leurs liaisons. Dans le cas de monocristaux elle est en général associée à la diffraction des électrons lents, ce qui permet d'avoir, en plus, des renseignements sur la structure de la surface.

Après un rappel de la désexcitation non radiative des atomes et des transitions Auger correspondantes, les problèmes que soulève l'interprétation des spectres d'énergie sont traités d'abord dans le cas d'un atome isolé puis dans celui d'un atome lié à des atomes voisins de même espèce ou d'espèces différentes.
\end{abstract}

On examine enfin quelques applications de l'analyse Auger.

Abstract. - Auger electron spectroscopy (A. E. S.) allows to characterize the atoms lying at surfaces of amorphous and crystalline materials and to investigate their chemical bond. In the case of single crystals it is generally associated with LEED which gives information on the surface structure.

After having recalled some principles about radiationless deexcitation of atoms and the related Auger transitions we discuss the (problems of) interpretation of energy spectra in the case of a single atom and their of an atom bound to neighbours either of the same kind or of a different one.

Some applications of A. E. S. as an analytical method are finally described.

1. Introduction. - Si l'effet Auger [1,2] a été mis en évidence en 1925 , ce n'est qu'après les travaux de Harris [3], Scheibner et Tharp [4] puis de Weber et Péria [5] en 1966 que la spectrométrie des électrons Auger proprement dite s'est développée. Cette méthode qui est devenue l'une des plus utilisées pour l'étude des surfaces [6-12] permet de caractériser les atomes situés à la surface d'un corps cristallin ou amorphe et de préciser la nature de leurs liaisons. Dans le cas d'échantillons monocristallins elle est très souvent associée à la diffraction des électrons lents, ce qui permet d'avoir en plus des renseignements sur la structure de la surface.

La très grande sensibilité de la méthode, qui permet de détecter une concentration de $10^{11}$ atomes $/ \mathrm{cm}^{2}$ (ce qui équivaut à $10^{-2}$ à $10^{-3}$ monocouche) et la faible profondeur d'émission des électrons (Fig. 13) fait que la gamme des applications de cette méthode est maintenant très étendue. Mais de nombreux problèmes d'ordre qualitatif et quantitatif se posent encore et nous avons essayé de les mettre en évidence à travers les exemples traités.

L'émission des électrons Auger peut être provoquée par divers rayonnements (RX, ions, électrons...) mais nous nous sommes intéressés dans cette étude essentiellement à l'excitation par faisceau d'électrons.

2. Considérations théoriques sur l'effet Auger. 2.1 MÉCANISME DE L'ÉJECTION D'UN ÉLECTRON AUGER. - Lorsqu'un atome isolé ou lié à d'autres atomes est ionisé, il existe essentiellement deux processus de relaxation. La relaxation peut être radiative (émission $\mathrm{X}$ ) ou non radiative (émission Auger).
Sur la figure 1 est représentée la probabilité de ces deux types de relaxation en fonction du numéro atomique $Z$ des éléments.

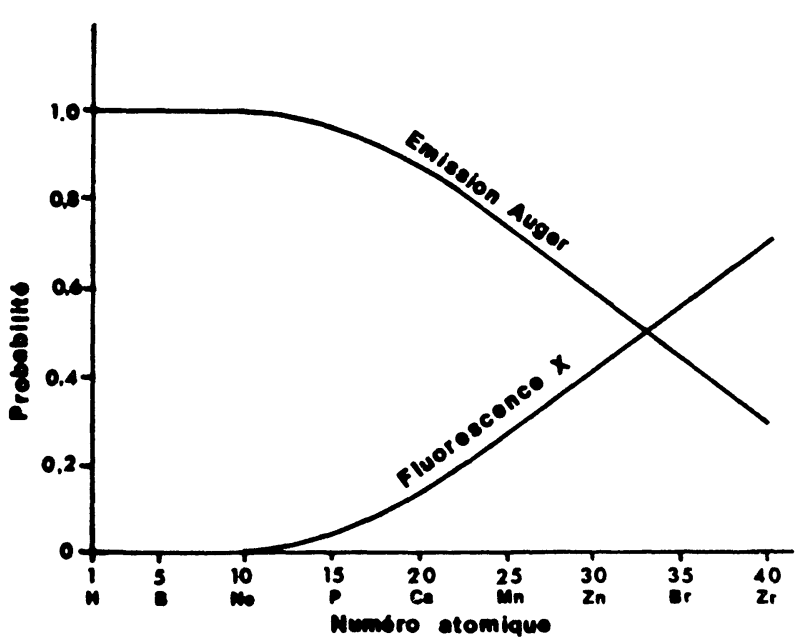

Fig. 1. - Rendement de l'émission Auger et de la fluorescence $\mathrm{X}$ en fonction du numéro atomique $Z$.

2.2 Relaxation non Radiative. - L'effet Auger est un processus de désexcitation non radiative d'un atome ionisé dans une couche $\mathrm{W}$. Ce processus se fait en deux temps.

- Remplissage de la lacune en $\mathrm{W}$ par un électron d'un niveau moins énergétique $X$; ce temps de relaxation est de $10^{-14}$ à $10^{-17} \mathrm{~s}$.

- Transfert de l'énergie $E_{\mathrm{W}}-E_{\mathrm{X}}$ à un électron d'un niveau $\mathrm{Y}$ tel que $\mathrm{E}_{\mathrm{Y}} \leqslant E_{\mathrm{W}}-E_{\mathrm{X}}$, qui sera éjecté 
dans le continuum. Cet électron, que nous appellerons électron Auger aura en première approximation une énergie :

$$
E_{\mathrm{A}}=E_{\mathrm{W}}-E_{\mathrm{X}}-E_{\mathrm{Y}} .
$$

Sur la figure 2 sont représentés les niveaux d'énergie d'un atome de silicium, ionisé au niveau $\mathrm{K}$.

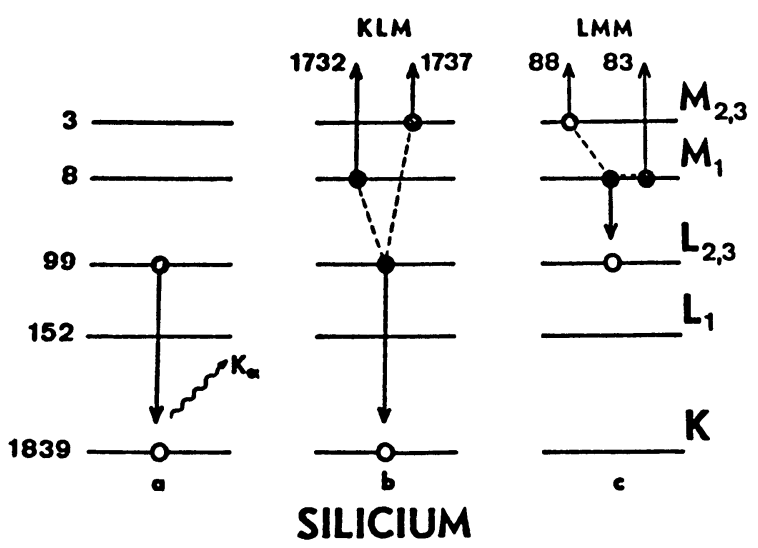

Fig. 2. $-a$ ) Désexcitation radiative; $b$ et $c$ ) désexcitation non radiative.

Cette énergie $E_{\mathrm{A}}$ mettant en jeu les énergies de trois niveaux de l'atome sera caractéristique de la nature de cet atome et de son état de liaison. La modification de l'état de liaison de l'atome entraîne un déplacement de ses niveaux d'énergie donc une variation de l'énergie de l'électron Auger. Il s'agit là d'un des apports importants de la méthode dans la mesure où les problèmes d'interprétation des spectres et en particulier des déplacements des pics $(\$ 4)$ peuvent être résolus.

2.3 Nomenclature des Électrons Auger. - Un électron Auger est décrit par les trois niveaux successivement mis en jeu. Dans le cas de la figure $2 b$ nous avons émission d'un électron KLM :

- la $1^{\text {re }}$ lettre désigne le niveau de la lacune initiale,

- la $2^{\mathbf{e}}$ lettre, l'origine de l'électron qui comble la lacune,

- la $3^{\mathrm{e}}$ lettre, le niveau de l'électron éjecté.

Dans le cas de la figure $2 c$ le niveau excité est $L$. Les électrons mis en jeu dans le processus Auger proviennent de la bande de valence. Nous appellerons cette transition LVV. L'énergie de ces électrons Auger LVV dépend plus particulièrement de la nature de la liaison chimique de l'atome.

Cette désignation des niveaux n'est rigoureusement correcte que si les interactions spin-orbite sont dominantes. Sur la figure 3 les énergies normées de transitions KLL sont tracées en fonction de $Z$.

Pour des $Z$ élevés le couplage spin-orbite est plus fort que l'interaction coulombienne; chaque lacune est définie par $l$ et $j$. Les deux lacunes couplent leurs $j$ et six transitions sont possibles. Pour des $Z$ faibles l'interaction coulombienne est forte ; c'est le couplage

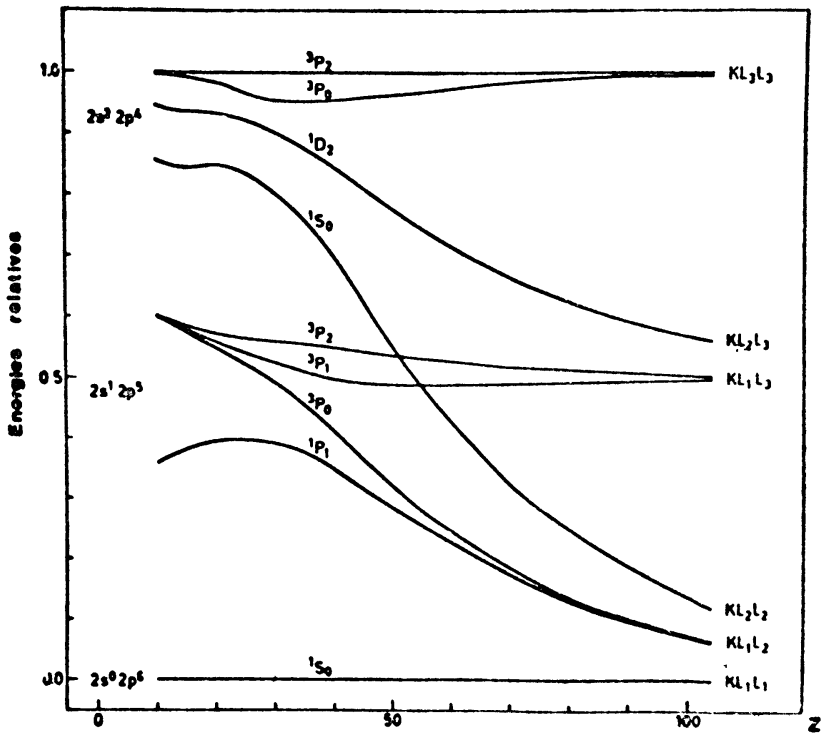

FIG. 3. - Energie relative des pics Auger KLL en fonction du numéro atomique $Z$.

$L-S$ (Russel Saunders) qui intervient et on utilise les termes spectroscopiques habituels pour désigner l'état final.

Pour des $Z$ intermédiaires des expériences à haute résolution (Hornfelt) [13] ont révèlé la présence de 9 lignes dans les spectres KLL ; dans ce cas la notation habituelle est complétée par la notation spectroscopique entre parenthèses.

3. Mise en évidence expérimentale des électrons Auger. - Si l'interprétation des spectres Auger pose des problèmes d'ordre théorique, elle est également très dépendante de l'appareillage et en particulier de sa résolution. On peut distinguer deux types d'analyseurs en énergie permettant la spectrométrie Auger :

- les appareils non dispersifs : à potentiel retardateur,

— les appareils dispersifs : spectromètres.

L'optique électronique d'un diffracteur d'électrons lents constitue le type principal d'appareil non dispersif et peut donc être utilisée pour la spectrométrie Auger.

Le potentiel retardateur $V_{\mathrm{r}}$ auquel on superpose une tension alternative $v$ est appliqué sur la grille $\mathbf{G}_{2}$ (Fig. 4), le courant ainsi filtré est collecté par l'écran et mesuré par un amplificateur à détection synchrone (Lock-in). Dans un appareil à 4 grilles on applique le potentiel retardateur sur les grilles $G_{2}$ et $G_{3}$. Le procédé de différentiation électrique utilisé permet d'obtenir directement la courbe de distribution de l'énergie $N(E)$ ou sa dérivée $\mathrm{d} N(E) / \mathrm{d} E$.

Il faut noter que très souvent la mise en évidence d'un pic Auger et sa mesure ne sont possibles que sur la courbe $\mathrm{d} N(E) / \mathrm{d} E$. En effet la suppression du fond continu qui en résulte permet de visualiser certains pics et facilite leur positionnement en énergie (Fig. 5). L'exploitation quantitative de l'effet Auger par contre ne peut se faire en toute rigueur que sur la courbe $N(E)$ (§ 6). 


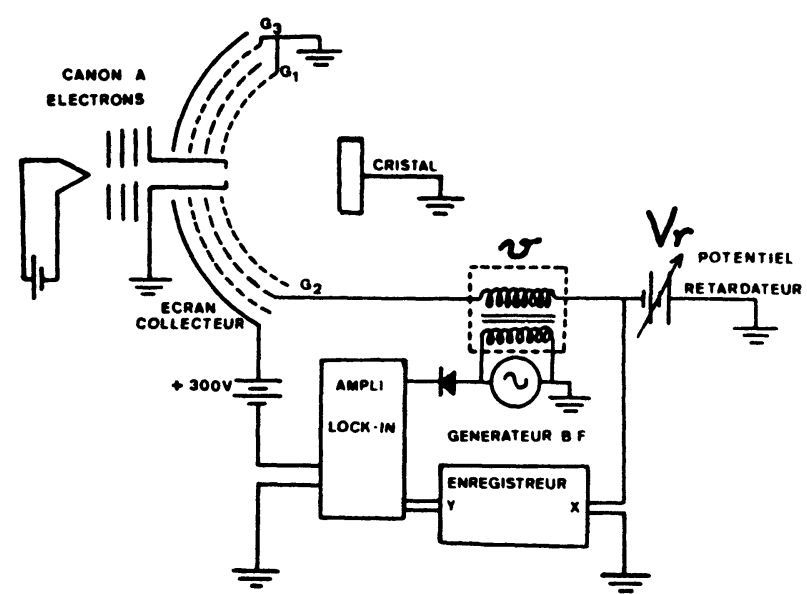

FIG. 4. - Schéma de l'optique électronique d'un diffracteur d'électrons lents utilisé en analyseur Auger à potentiel retardateur.

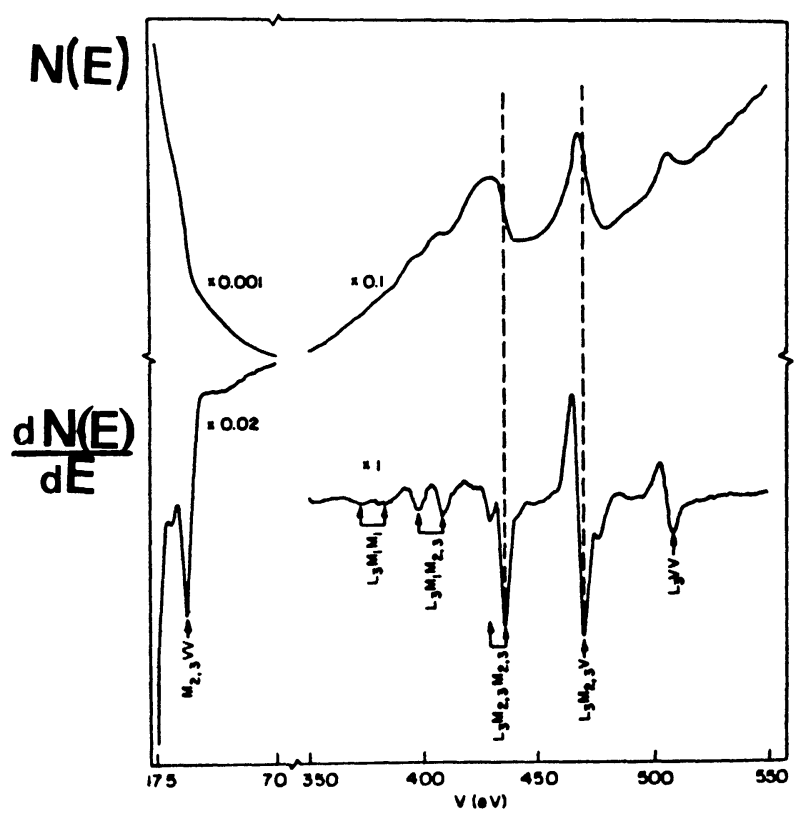

Fig. 5. - Spectre Auger du vanadium.
La résolution $\Delta E / E$ du dispositif à potentiel retardateur est de l'ordre de $1 / 100$ ce qui peut paraître suffisant si on rappelle que la déconvolution du pic Auger fait intervenir 3 niveaux ; toutefois dans certains cas les pics Auger de deux éléments (ex. Pt et S) peuvent se chevaucher. Une meilleure résolution permet souvent leur déconvolution. L'analyseur dispersif à miroir cylindrique (C. M. A.) (Fig. 6) a une résolution de l'ordre de $1 / 500$; équipé d'autre part d'un multiplicateur d'électrons, il permet d'utiliser la technique du comptage et aura donc une plus grande sensibilité. Cette qualité est importante en analyse Auger quantitative (§ 6), le traitement du signal impulsionnel permettant notamment de restituer une courbe $N(E)$ dont la surface des pics Auger peut être exploitée.

4. Interprétation des spectres Auger. - Les transitions Auger telles qu'elles viennent d'être décrites sont en fait beaucoup plus compliquées car le passage de l'atome isolé au solide et l'existence des transitions de Coster-Kronig modifient les données simplifiées présentées en 2.2.

4.1 Passage de l'Atome isolé Au Solide. - Quand on passe de l'atome isolé à l'état solide les niveaux d'énergie s'élargissent en bandes (Fig. 7a). Ce sera en particulier le cas pour la bande de valence.

4.2 Transitions De Coster-Kronig. - Des transitions Auger d'un type particulier appelées CosterKronig peuvent apparaître. Il s'agit d'un processus extrêmement rapide qui revient à déplacer la lacune initiale vers une sous-couche supérieure. Avant même d'être désexcité par un processus Auger, l'atome se trouve donc doublement ionisé et les énergies des électrons s'en trouvent affectées. Ces transitions Coster-Kronig du type $\mathrm{L}_{i} \mathrm{~L}_{j} \mathrm{X}$ ou $\mathrm{M}_{i} \mathrm{M}_{j} \mathrm{X}$ sont très intenses.

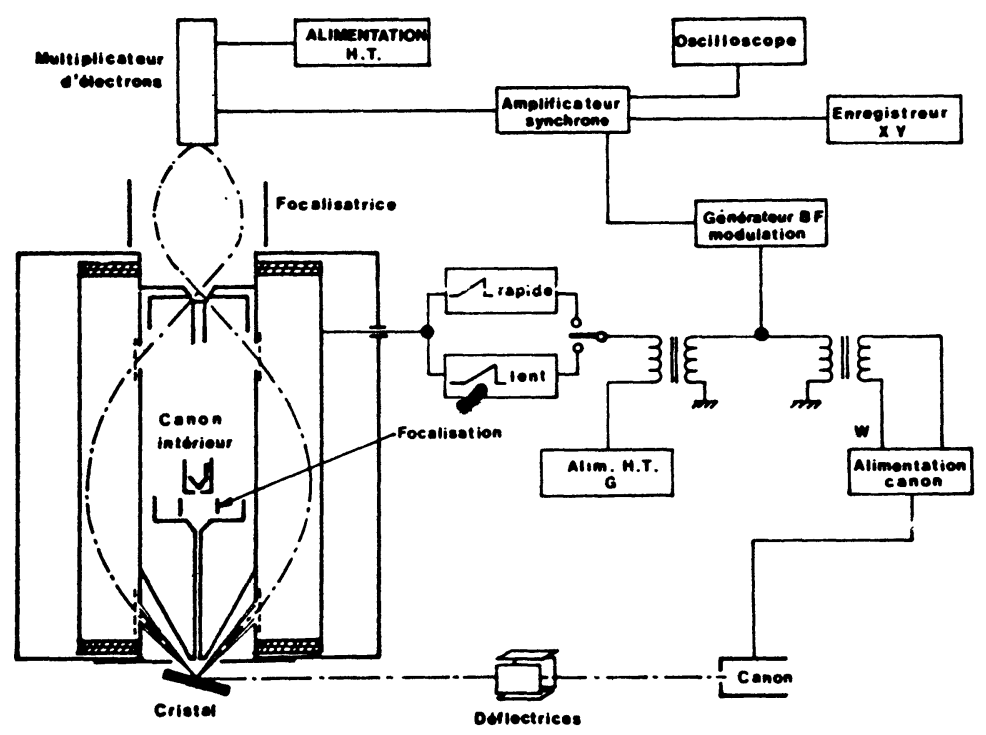

Fig. 6. - Schéma électrique d'un spectromètre Auger à miroir cylindrique (C. M. A. = Cylindrical Miror Analyser). 

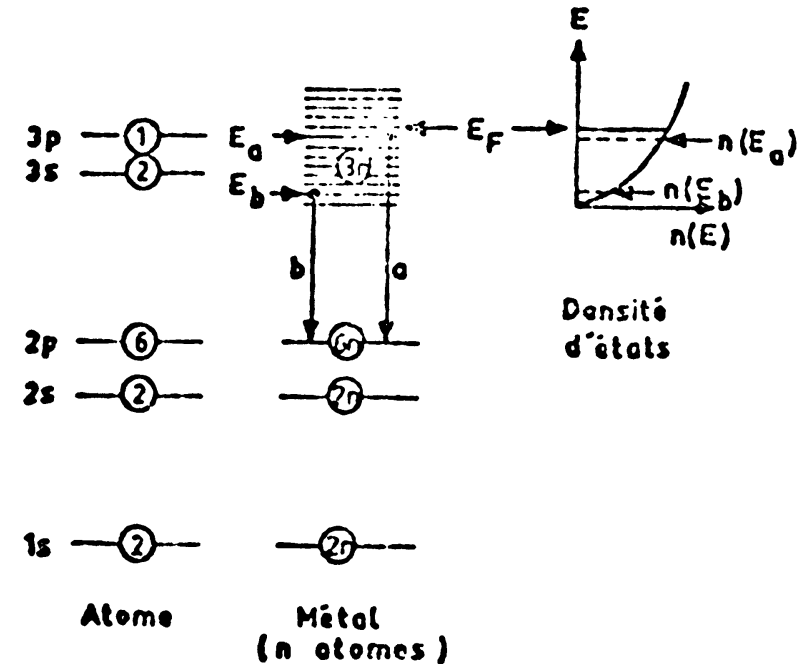

Fig. 7. - Niveaux d'énergie $a$ ) dans un atome isolé, $b$ ) dans un métal.

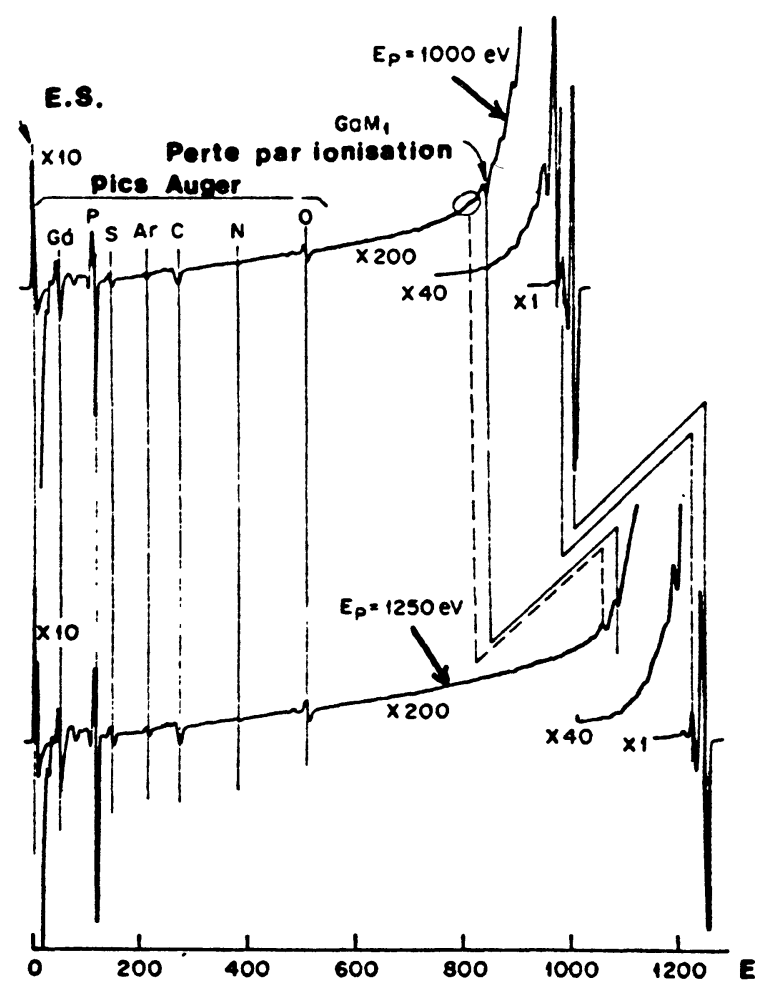

Fig. 8. - Spectres Auger du GaP pour deux énergies primaires différentes.

4.3 INFLUENCE DE L'ÉNERGIE ET DE L'ANGLE D'INCIDENCE DU FAISCEAU D'EXCITATION. - La section efficace d'ionisation d'un atome Bourdon donc l'intensité des pics Auger varie peu avec l'énergie du faisceau d'excitation (Fig. 7b). On admet que cette section efficace est maximum pour $E_{\mathrm{p}}=3 E_{\mathrm{W}}$. Le rendement Auger varie par contre avec l'angle d'incidence 0 . Ce fait est à relier à l'augmentation du volume irradié qui varie approximativement comme $1 / \cos \theta$. Ce rendement est maximum si $\theta$ est voisin de $90^{\circ}$.
5. Energie d'une transition Auger. - Nous avons vu en 2 que l'énergie d'une transition Auger était en première approximation $E_{\mathrm{W}}-E_{\mathrm{X}}-E_{\mathrm{Y}}$. Ces énergies $E_{i}$ correspondent à l'atome non ionisé, or l'énergie $E_{\mathrm{W}}-E_{\mathrm{X}}$ est transférée à un électron du niveau Y d'un atome déjà ionisé. On peut penser que tout se passe comme si on était en présence de l'ionisation du niveau $\mathrm{Y}$ d'un atome de numéro atomique $Z+1$. Nous devons d'autre part tenir compte du potentiel de contact $\varphi_{\mathrm{A}}$ de l'analyseur. L'énergie de l'électron Auger peut alors s'écrire :

$$
\begin{gathered}
E_{\mathrm{A}}=E_{\mathrm{W}}(Z)-E_{\mathrm{X}}(Z)-E_{\mathrm{Y}}(Z+1)-\varphi_{\mathrm{A}} \\
\text { (formule de Burhop [6]) }
\end{gathered}
$$

En fait cette formule est encore approchée surtout lorsque les niveaux de valence sont mis en jeu et on a été conduit à chercher d'autres formules empiriques qui donnent des résultats plus proches de l'expérience. La formule de Chung et Jenkins [14] est actuellement une des plus utilisées :

$$
\begin{aligned}
E_{\mathrm{A}}= & E_{\mathrm{W}}(Z)-\frac{1}{2} E_{\mathrm{X}}(Z)+ \\
& +E_{\mathrm{X}}(Z+1)-\frac{1}{2} E_{\mathrm{Y}}(Z)+E_{\mathrm{Y}}(Z+1)-\varphi_{\mathrm{A}} .
\end{aligned}
$$

Du point de vue théorique peu de calculs sont disponibles et il faut signaler les différences importantes qui existent entre théorie et expérience pour le moment. Ces calculs font intervenir les modes de couplage entre orbitales qui décrivent les lacunes de l'état final. Pour les $Z$ faibles c'est le couplage $L-S$ de Russel et Saunders ; pour les $Z$ grands c'est le couplage $J$-j qui intervient ; il faut d'autre part tenir compte de l'énergie de relaxation (Shirley) [15]. De tels calculs deviennent fort compliqués pour des transitions du type LLM, LMM etc...

5.1 ASPECT QUALITATIF. - Exploiter qualitativement les spectres Auger revient à pouvoir indexer les pics et à interpréter leurs déplacements. L'utilisation de formules telles que celles de Chung et Jenkins est souvent suffisante pour caractériser des échantillons conducteurs formés d'un seul type d'atomes, ainsi que leur contamination superficielle.

L'existence de plusieurs transitions caractéristiques d'un même élément, l'utilisation de tables donnant les énergies de liaison pour les niveaux de l'atome (table de Bearden et Burr) [16] et l'apport de l'XPS $\left({ }^{1}\right)$ ont permis à ce jour d'établir des catalogues de spectres très complets [8], [17].

Les figures $9 a$ et $9 b$ montrent deux spectres de fer dopé au bore [18] l'un sans traitement, l'autre après un chauffage à $1050^{\circ} \mathrm{C}$. Les pics qui correspondent aux différentes transitions Auger sont bien séparés et il n'y a aucune ambiguïté.

Le problème est plus complexe quand l'existence d'atomes voisins provoquent des déplacements chimiques des niveaux de l'atome considéré et modifie l'énergie des pics Auger.

(1) XPS : X-ray Photoelectron Spectroscopy. 


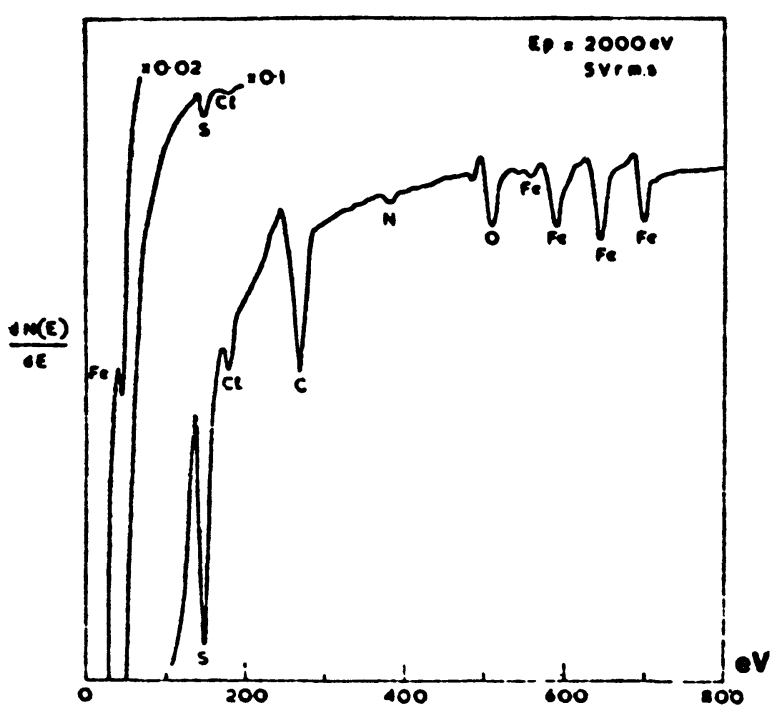

Fig. $9 a$. - Spectre Auger du fer dopé au bore avant traitement thermique.

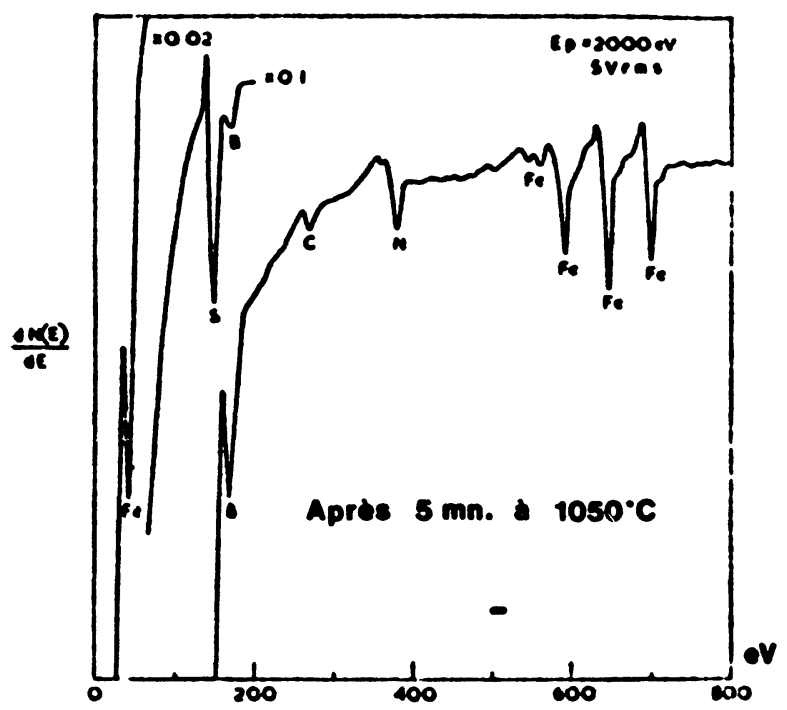

FIG. $9 b$. - Spectre Auger du fer dopé au bore après traitement thermique.

Si nous considérons les spectres de la figure $10 \mathrm{du}$ silicium dans le métalloïde et dans un verre du type $\mathrm{Na}_{2} \mathrm{O}, \mathrm{SiO}_{2}[19]$ nous voyons que non seulement le pic

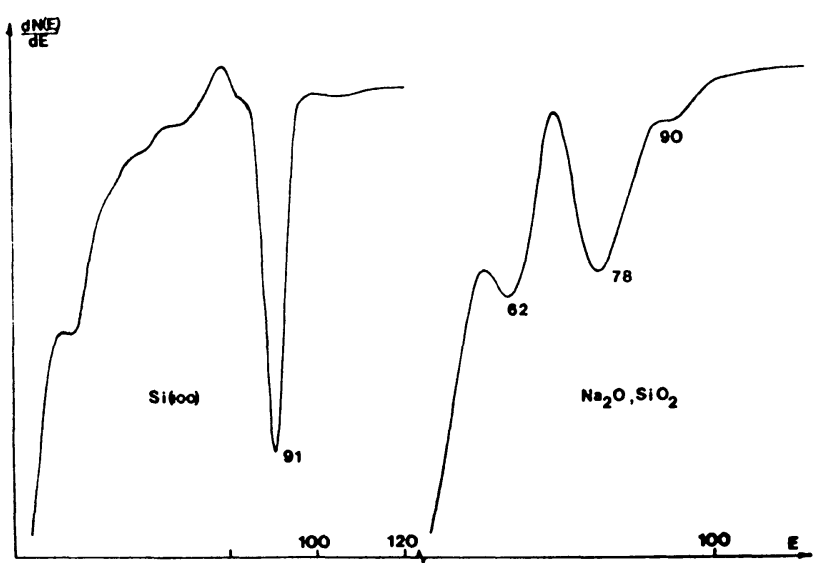

Fig. 10. - Spectre Auger du silicium et d'un verre $\mathrm{Na}_{2} \mathrm{O}, \mathrm{SiO}_{2}$. est décalé en énergie ( $12 \mathrm{à} 13 \mathrm{eV}$ pour la transition $\mathrm{L}_{2,3} \mathrm{VV}$ du silicium) mais encore que la forme est fonction de la nature de la liaison de l'atome. Cet élargissement est susceptible de fournir des renseignements intéressants mais on conçoit aussi que de sérieux problèmes de déconvolution peuvent alors se poser.

Un autre exemple est donné par Grant et Haas [20] (Fig. 11). Le pic du carbone a une forme et une position différentes dans divers composés.

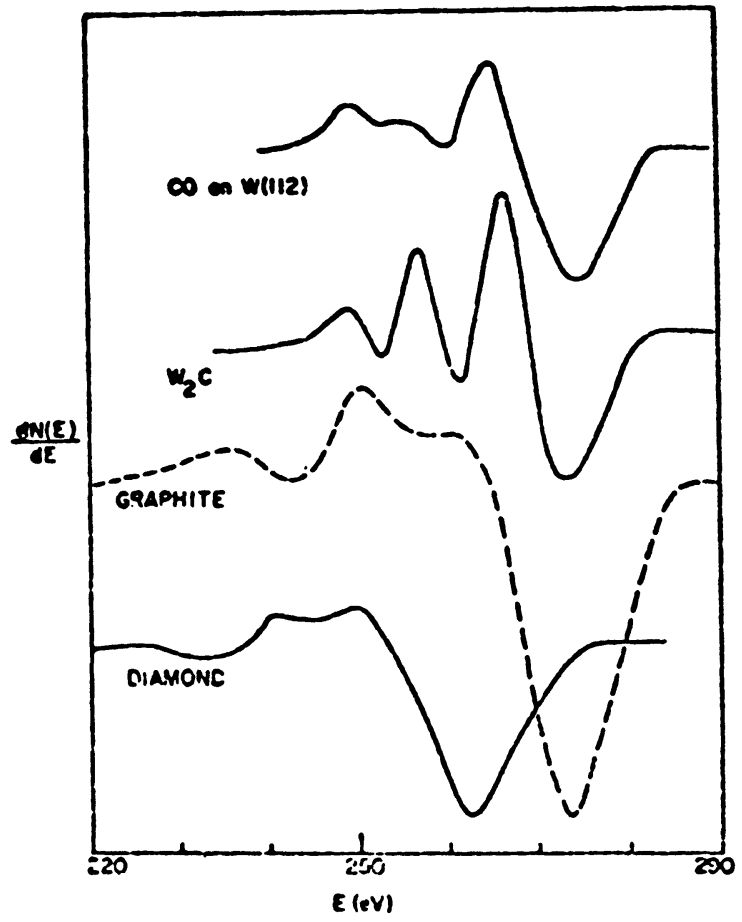

Fig. 11. - Spectre Auger du carbone dans différents composés.

Cet exemple montre également qu'il est important de disposer d'un spectromètre ayant une bonne résolution en énergie afin de pouvoir mettre en évidence de faibles déplacements des pics Auger.

L'interprétation est particulièrement délicate si l'échantillon est un isolant. Aux déplacements en énergie dus à l'environnement chimique s'ajoutent des déplacements dus aux phénomènes de charges. Un exemple intéressant est celui de l'étude du $\mathrm{MgO}$ par l'équipe de Prutton [21]. On constate (Fig. 12) un déplacement très important en énergie du pic du Magnésium par rapport à sa valeur dans le métal, déplacement d'autant plus grand que le $\cos \theta$ est petit. Ce déplacement s'interprète comme résultant d'une part de la charge de la surface Bourdon qui est fonction de $\cos \theta$ et d'autre part de l'environnement du magnésium dans l'oxyde.

Nous avons pu faire des constatations identiques au cours de nos études des surfaces de silice vitreuse et de silicates [22].

5.2 Mesure De LA DENSité D'etaTs-STRUCTURE DE BANDE. - Il est important de connaître le nombre d'états électroniques dans les niveaux externes car 

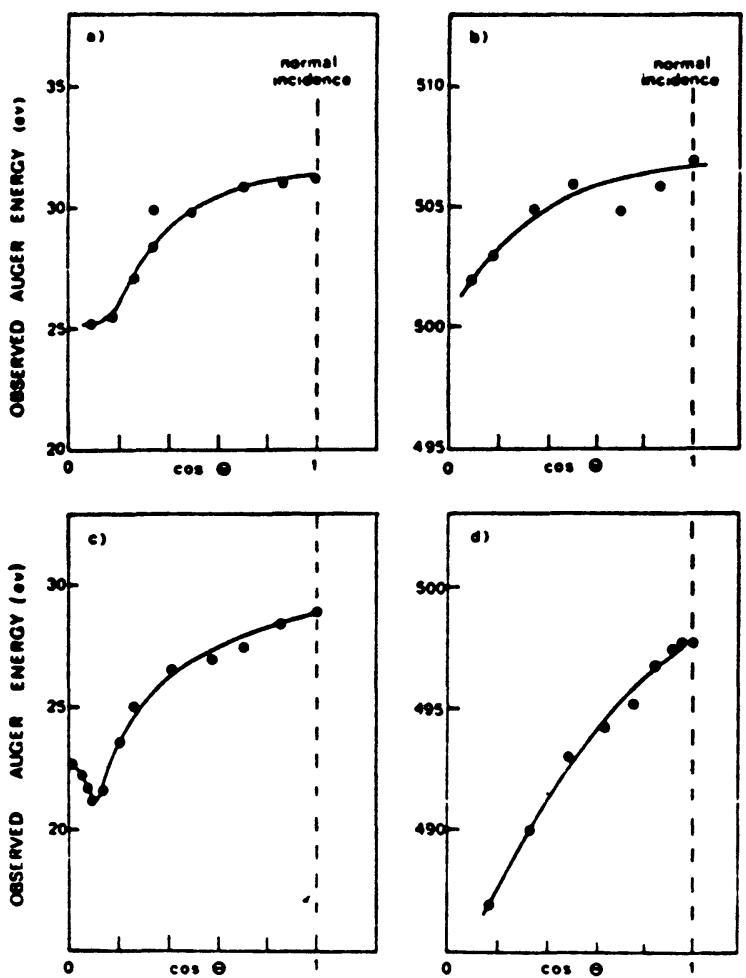

FIG. 12. - Analyse Auger du magnésium oxydé et de l'oxyde de magnésium : variation de la position du pic Auger avec l'angle d'incidence : a) Pic du magnésium dans le magnésium oxydé,

b) Pic de l'oxygène dans le magnésium oxydé, c) Pic du magnésium dans la magnésie, $d$ ) Pic de l'oxygène dans la magnésie.

beaucoup de propriétés en dépendent. Les surfaces des solides présentent des états électroniques localisés qu'on appelle états de surface.

L'existence de ces états de surface est une conséquence de l'interruption du réseau par la surface. C'est Heine [23] qui a traité le problème du point de vue général. Du point de vue expérimental il s'agit donc d'avoir une information sur la répartition des états dans la bande de valence. Salmeron, Baro et Roja [24] du laboratoire de Cabrera à Madrid ont montré qu'il était possible d'utiliser la spectrométrie des électrons Auger pour déterminer la distribution des densités d'états du cuivre. Mais il faut noter que l'AES( $\left.{ }^{2}\right)$ n'est pas la technique idéale pour de telles études ; en effet l'information que nous recueillons c'est $N(E)$. Or cette distribution d'énergie $N(E)$ est le produit de convolution des distributions d'états de 3 niveaux WXY qui définissent la transition Auger. L'opération de déconvolution est en général très délicate. On peut citer également un travail récent de Arnott et Haneman [25] qui a étudié la structure de la bande de valence du silicium.

6. Intensité d'un pic Auger. - L'intensité d'une transition Auger est donnée par la relation suivante :

$$
I=v \frac{P_{\mathrm{Ai}}}{\sum P_{\mathrm{Ai}}}
$$

$v$ est la vitesse de formation d'une lacune, $P_{\mathrm{Ai}}$ probabilité d'une transition Auger particulière, $\sum P_{\mathrm{Ai}}$ probabilité totale de désexcitation d'une lacune.

Cette intensité est donc proportionnelle au nombre de lacunes créées, donc en première approximation au nombre d'atomes présents ; l'étude quantitative peut donc être envisagée.

Le nombre de lacunes dans une couche est proportionnel à la population initiale du niveau, mais le processus de Coster-Kronig peut considérablement modifier cette densité de lacunes. Ainsi Mac Guire a montré que pour l'élément $Z=42$, la transition de CosterKronig met en mouvement $86 \%$ des trous de $L_{1}$ vers $L_{2}$ et $\mathrm{L}_{3}$. Si la distribution initiale des lacunes est proportionnelle à 2,2 et 4 elle devient proportionnelle à 1,7 et 16. Cette modification dans la distribution des lacunes

(2) AES : Auger Electron Spectroscopy.

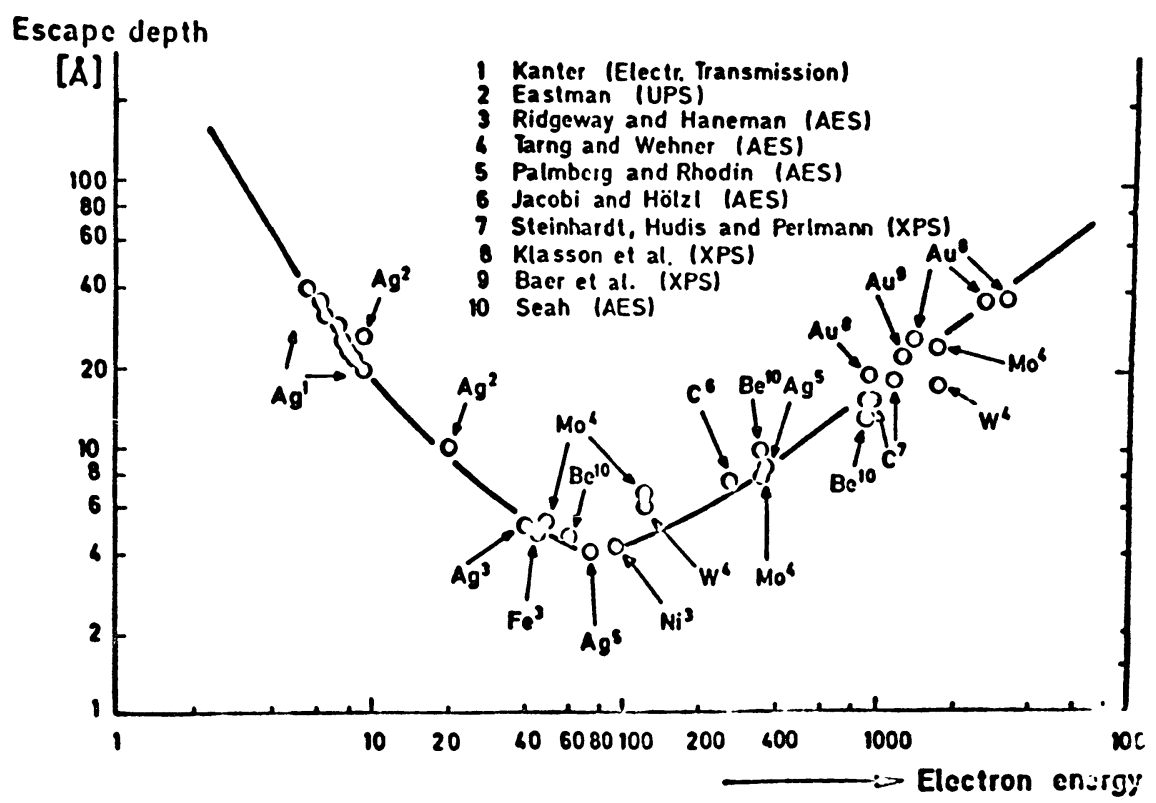

FIG. 13. - Variation du libre parcours moyen des électrons en fonction de l'énergie. 
introduit des erreurs notables en analyse Auger quantitative. Il faut noter enfin que le libre parcours moyen des électrons est fonction de leur énergie (Fig. 13), le volume de l'échantillon concerné peut donc être très différent pour certains éléments. De ce fait l'interprétation quantitative des spectres Auger se complique.

6.1 ASPECT QUANTITATIF. - L'étude quantitative ne devrait être effectuée en toute rigueur que sur les courbes $N(E)$ pour lesquelles la surface des pics est proportionnelle au nombre d'atomes présents. Comme on dispose en général des courbes $\mathrm{d} N(E) / \mathrm{d} E$ des procédés d'intégration numérique sont nécessaires ce qui demande un appareillage analyseur très sensible travaillant en comptage. Aussi de nombreuses études ont-elles été effectuées en mesurant la hauteur du pic $\mathrm{d} N(E) / \mathrm{d} E$. Un étalonnage avec une autre méthode est nécessaire dans ce cas.

On a pu ainsi combiner l'ellipsométrie à l'AES. Meyer et Vrakking [25] ont étudié l'adsorption d'oxygène sur $\mathrm{Si}$ (111) et ont obtenu une relation linéaire entre la hauteur du pic Auger et le paramètre d'ellipsométrie $\Delta \delta$ qui est proportionnel au taux de recouvrement entre 0 et 1 monocouche.

Une autre méthode consiste à utiliser des traceurs radioactifs. Cette technique a été développée par Oudar et Perdereau [26] pour doser le soufre. Des méthodes utilisant des étalons internes sont également possibles.

Récemment enfin C. C. Chang [27] a proposé un formalisme intéressant pour l'étude quantitative.

\subsection{ETUDE DE LA DISTRIBUTION ANGULAIRE DES} ÉleCtrons Auger. DÉTERMINATION DE LA COMPOSITION EN SURFACE DES ALLIAGES. - L'intérêt des structures électroniques des alliages et leur utilisation de plus en plus fréquente en catalyse a amené le physicochimiste à s'intéresser à la mesure de leur composition en surface. Des arguments simples de thermodynamique permettent de prévoir une composition de la surface très différente de celle du volume [28]. L'étude de la distribution angulaire des électrons Auger permet de mesurer la composition en surface d'un alliage.

Christmann et Ertl [29] ont étudié le système ArgentPalladium. L'argent et le palladium sont miscibles en toutes proportions et la loi de Vegart [30] est applicable, ce qui permet d'évaluer la composition en volume de l'alliage à partir des diagrammes de diffraction d'électrons rapides.

Sur la figure 14 sont représentés les spectres Auger obtenus respectivement sur une surface de palladium, d'un alliage $\mathrm{Pd} / \mathrm{Ag}(22 \% \mathrm{Ag})$ et d'argent.

Pour étudier la variation de la composition de l'alliage dans les premières couches atomiques on peut étudier la hauteur des pics en fonction de l'angle $\alpha$ que forme le faisceau d'électrons incidents avec la surface du cristal. Si on admet que la surface est bien lisse les électrons Auger proviennent d'une profondeur $d=1 \sin \alpha ; l$ est le libre parcours moyen des électrons primaires

$$
\left(l=30 \AA \text { pour } E_{\mathrm{p}}=2000 \mathrm{eV}\right) .
$$
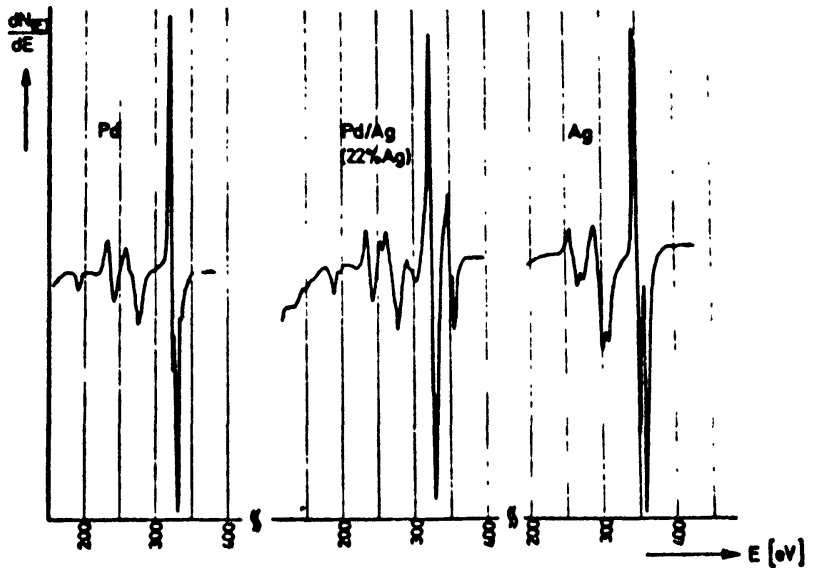

FIG. 14. - Spectres Auger du palladium, de l'alliage Pd/Ag et de l'argent.

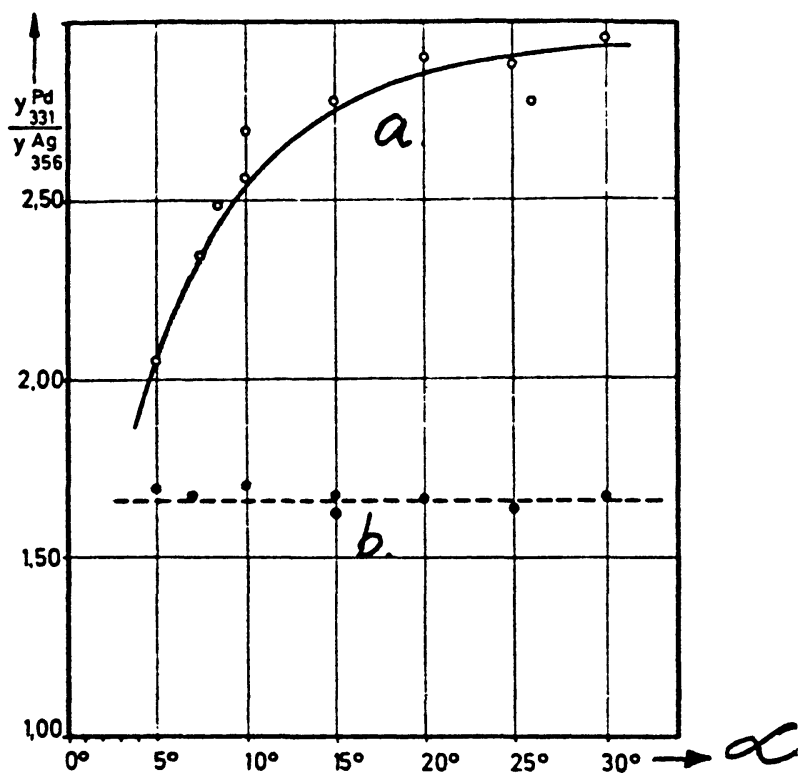

Fig. 15. - Variation du rapport $y^{\mathrm{Pd}} / y^{\mathrm{Ag}}$ en fonction de l'angle : $a$ ) cas de l'alliage non homogène; $b$ ) cas de l'alliage homogène.

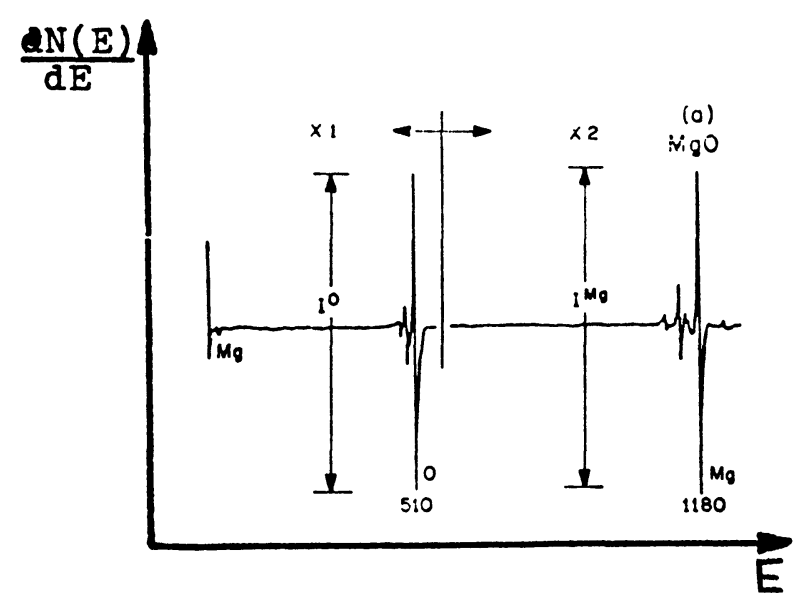

FIG. 16. - Spectre Auger obtenu sur une surface de magnésie par excitation $\mathrm{X}$. 
Ainsi pour $\alpha=10^{\circ}$ la profondeur concernée est de $5 \AA$ A. Sur la figure 15 est représenté le rapport $y^{\mathrm{Pd}} / y^{\mathrm{Ag}}$ en fonction de l'angle $\alpha$. ( $y^{\mathbf{P d}}, y^{\mathbf{A g}}$ sont les hauteurs des pics Auger du palladium et de l'argent.)

La courbe de la figure $15 a$ montre un enrichissement en argent de la surface ; la courbe de la figure $15 b$ est caractéristique d'un alliage bien homogène.

7. Comparaison entre les spectres Auger excités par les électrons et ceux excités par les RX. - Dans les deux cas on obtient des spectres Auger assez comparables. Il existe toutefois certaines différences :

D'une étude de Fiermans et al. [31] il ressort que les

différences dans les déplacements observés proviennent d'un changement d'énergie de relaxation.

On peut noter d'autre part que certaines différences d'énergie peuvent provenir d'effets de charge. Ainsi la courbe de la figure 16 montre un spectre Auger obtenu par Wagner et al. [32] sur une surface de MgO par excitation $\mathrm{X}$.

Si on compare ce spectre à celui obtenu par Prutton [21] sur le $\mathrm{MgO}$ mais par excitation électronique on peut noter une différence notable entre les énergies du pic Auger de Mg dans les deux cas. Dans le tableau suivant les résultats de ces deux expériences ont été comparés aux valeurs calculées (Fig. 17).

$\begin{array}{cccc}\begin{array}{c}\text { Transition } \\ \text { Auger }\end{array} & \begin{array}{c}\mathrm{a} \\ \text { (Prutton) }\end{array} & \begin{array}{c}\mathrm{b} \\ \text { (Wagner) }\end{array} & \begin{array}{c}\mathrm{c} \\ \text { (Chung) }\end{array} \\ \mathrm{KL}_{23} \mathrm{~L}_{23} & - & - & - \\ \mathrm{KL}, \mathrm{L}_{23} & 1168 \mathrm{eV} & 1180 \mathrm{eV} & 1181 \mathrm{eV} \\ & 1124 \mathrm{eV} & 1134 \mathrm{eV} & 1139 \mathrm{eV}\end{array}$

FIg. 17. - Comparaison de l'énergie des pics Auger du Mg obtenus par excitation électronique (a), et par excitation $X(b)$ aux valeurs calculées (c).

8. Conclusion. - La spectrométrie des électrons Auger a connu ces dernières années un grand développement ; les quelques exemples traités illustrent l'importance de cette technique pour l'étude des surfaces, qu'il s'agisse de déterminer la nature des éléments, leur nombre ou leur état électronique. Cette méthode s'applique aux domaines les plus variés : Métallurgie, Chimie (notamment la catalyse) et Physique du solide.

Il ne faut toutefois pas passer sous silence les nombreuses difficultés qui subsistent encore actuellement notamment dans l'interprétation des spectres. Ce n'est très souvent que grâce à la convergence de résultats provenant de techniques différentes qu'une conclusion peut être tirée sans ambiguïté. L'appareil idéal du physico-chimiste des surfaces de demain devrait donc comporter les accessoires suivants : LEED $\left({ }^{3}\right)$, AES, SIMS $\left({ }^{4}\right)$, UPS $\left({ }^{5}\right)$ et XPS.

(3) LEED = Low Energy Electron Diffraction.

(4) SIMS = Secondary Ions Mass Spectroscopy.

(5) UPS = Ultra-violet Photoelectrons Spectroscopy.

\section{Bibliographie}

[1] Auger, P., J. Physique Rad. 6 (1925) 205.

[2] Auger, P., Surf. Sci. 47 (1975) 1.

[3] Harris, L. A., J. Appl. Phys. 39 (1968) 1419.

[4] Scheibner, E. J. et Tharp, L. N., Surf. Sci. 8 (1967) 247.

[5] Weber, R. E. et Peria, W. T., J. Appl. Phys. 38 (1967) 4355.

[6] Burhop, E. H. S., The Auger effect and other radiationsless transitions (Cambridge University Press) 1952.

[7] Deville, J. P., Revue Phys. Appl. 3 (1968) 351.

[8] Palmberg, P. W. et al., Handbook of Auger Electron Spectroscopy (Physical Electronics Inc.) 1972.

[9] Tracy, J. C., Proceedings of the Nato Sommer Institute held at the University of Gent, Belgium 1972.

[10] Somorjai, G. A., Principles of Surface Chemistry, Prentice Hall Inc., 1972.

[11] Riwan, R., Méthodes d'études des interactions GazSurface Centre d'actualisation scientifique et technique, I. N. S. A., Lyon, 1973.

[12] Deville, J. P., Séminaire de Physique des surfaces, Strasbourg, 1975.

[13] HORNFELT, Ark. Fys. 155 (1962).
[14] Chung, M. F. et Jenkins, L. H., Surf. Sci. 22 (1970) 479. [15] Shirley, D. A., Phys. Rev. A 7 (1973) 1520, Chem. Phys. Lett. 16 (1972) 220.

[16] Bearden, J. A. et Burr, A. F., Rev. Mod. Phys. 37 (1967) 128.

[17] Coghlan, W. A. and Clausing, R. E., Catalog of calculated Auger transition for the elements USAEC Report ORNL-TM-3576 Oak Ridge National Laboratory, Surf. Sci. 33 (1972) 411.

[18] Bishop, H. E. et Rivière, J. C., Acta Met. 18 (1970) 813.

[19] Carrière, B., Deville, J. P. et Goldsztaub, S., Silicates Industriels 11 (1974) 313.

[20] Grant, J. T. and HaAs, T. W., Surf. Sci. 24 (1971) 332; Haas, T. W. and Grant, J. T., Appl. Phys. Lett. 16 (1970) 172.

[21] Janssen, A. P., Schoonmaker, R. C., Chambers, A. and Prutton, M., Surf. Sci. 45 (1974) 45.

[22] Goldsztaub, S., Burggraf, C., Carrière, B. et Deville, J. P., Communication présentée à la «Condensed Matter Division of the European Physical Society », Budapest, octobre 1974. 
[23] Heine, V., Proc. Phys. Soc. 81 (1963) 300.

[24] Salmeron, M., Baro, A. M. and Roja, J. M. (à paraître dans Surf. Sci.).

[25] Meyer, F. and Vrakking, J. J., Surf. Sci. 33 (1972) 271.

[26] Perdereau, M., Surf. Sci. 24 (1971) 239.

[27] Chang, C. C., Surf. Sci. 48 (1975) 9.
[28] Overbury, S., Bertrand, P. A. and Somorjai, G. A., LBL, Report 2746 (1974).

[29] Christmann, K. and ErtL, G., Surf. Sci. 33 (1972) 254.

[30] Moss, R. L. and Thomas, D. H., Trans. Faraday Soc. 60 (1964) 1110.

[31] Fiermans, L., Hoogewis, R. and Vennix, J., Surf. Sci. 47 (1975) 1.

[32] Wagner, C. D. and Biloen, P., Surf. Sci. 35 (1973) 82. 